\title{
Suspending Medical Student Clerkships Due to COVID-19
}

\author{
Matthew N. Goldenberg ${ }^{1}$ (D) - David C. Hersh ${ }^{2} \cdot$ Kirsten M. Wilkins ${ }^{1} \cdot$ Michael L. Schwartz ${ }^{3}$
}

Published online: 3 June 2020

(C) International Association of Medical Science Educators 2020

\begin{abstract}
Medical schools around the world have been grappling with how to adapt undergraduate medical curricula in the face of the COVID-19 pandemic. Our institution made the decision to suspend all clinical clerkships the day before the Association of American Medical Colleges (AAMC) recommended to US medical schools a similar suspension of "medical student participation in any activities that involved patient contact." This manuscript describes the rapid evolution in our decision-making as we weighed various information, values, and priorities in the face of the emerging public health crisis. We discuss how a compromised learning environment and concerns about student, patient, and the public health led to the suspension. We also consider next steps as we move forward in this uncertain time.
\end{abstract}

Keywords Medical students $\cdot$ Clinical clerkship $\cdot$ Medical school administration $\cdot$ COVID-19

The COVID-19 pandemic has disrupted virtually every segment of the American society, including medical education. On March 17, in an unprecedented decision, the Association of American Medical Colleges (AAMC) recommended that all US medical schools suspend clinical clerkships for at least 2 weeks [1]. Like peers around the country and the world, educational leaders at our institution, the Yale University School of Medicine, had been grappling with how to adapt our undergraduate medical curriculum in the face of the pandemic. We had already suspended clinical clerkships the day prior to the AAMC's recommendation, a decision that we had not at all anticipated just 2 weeks before.

\section{History}

The emerging SARS-CoV-2 threat and its potential impact on medical student education was a featured item on the agenda of

Matthew N. Goldenberg

matthew.goldenberg@yale.edu

1 Department of Psychiatry, Yale University School of Medicine, New Haven, CT, USA

2 Department of Pediatrics, Yale University School of Medicine, New Haven, CT, USA

3 Office of Education, Yale University School of Medicine, New Haven, CT, USA the regularly scheduled monthly meeting of Clerkship Directors (CDs) on March 3. Our students begin their clinical clerkships in January of their second year, so at the time the current clerkship students were about 8 weeks into their first 12 -week clerkship. Though no COVID-19 cases had yet been reported in Connecticut, the Yale-New Haven Health system and our academic center were making extensive preparations for the likelihood that we would be evaluating and treating patients with the virus in the near future. As part of these preparations, the CDs, students, and faculty alike had begun to question whether and how trainees would be involved in the care of these patients. CDs discussed what our response should be in terms of policies, processes, and messaging to students.

CDs agreed that caring for the sick, particularly in difficult times, is a professional responsibility and that it is important that medical students witness and participate in such care. Medical students in academic health centers often have more time than their supervising physicians to spend with individual patients and to think about their diagnosis and treatment. We anticipated that being in hospitals and clinics during a time of crisis preparation and response could provide particularly good learning opportunities. Students on many services would surely learn about the symptoms, diagnosis, and treatment of COVID-19. All students would also have an opportunity to view the physician role in public health including policy development and public messaging. Emerging stories of racial profiling and discrimination against Asians during the outbreak would provide a stimulus for students to consider issues of race and racism in medicine. 
We discussed that preliminary epidemiologic evidence from China and other countries suggested that people in their 20 s and 30 s (the age of nearly all our students) without pre-existing health conditions seemed to be at minimal risk for severe disease from SARS-CoV-2. We agreed that students with personal circumstances that might put them or loved ones at higher risk could self-identify to the Associate Dean for Student Affairs, and alternative arrangements could be explored.

Some caveats were mentioned at that meeting. CDs readily agreed that students would only be expected to perform tasks appropriate to their training level. Though no specifics were defined at the time, we felt limits could and should be placed on what aspects of care clerkship students could provide patients with COVID-19. We analogized any future restrictions to those already in place for learners (e.g., clerkship students are not permitted to perform invasive procedures such as drawing blood on patients who are HIV positive during their first 6 months on clerkships). At the beginning of March, conservation of personal protective equipment (PPE) was beginning to be discussed by hospital leadership, but there was no prohibition regarding the students' use of PPE.

On March 6, the Dean of the medical school issued a statement via email to the school community, affirming the role of students on clerkships, electives, and sub-internships as "essential to the academic medical center's patient care mission."

Over the next several days, many more cases of COVID-19 were reported nationally and the outbreak became more locally apparent. Calls for social distancing were increasing; academic conferences were cancelled; professional and personal travel restrictions were recommended; our university issued a policy that activities with groups of greater than 100 participants should be cancelled. The pre-clinical curriculum at our medical school was quickly moved to online platforms in order to facilitate social distancing among students.

CDs came together again via teleconference in an ad hoc meeting on March 10 to discuss the developing situation. We reaffirmed our original contention that caring for infected patients is a professional duty of both physicians and physicians-to-be. We agreed to try to move many of our didactic sessions to online platforms when possible and to limit group sizes in any classroom or ward activity to 10 or less people. Some CDs raised concern that such restriction might marginalize students at events like joint resident-student didactic conferences in the hospital.

Following that meeting, the Deputy Dean for Education, the Associate Dean for Curriculum, the Associate Dean for Student Affairs, and the Director of Clerkships emailed students regarding their role in the care of patients suspected of or confirmed to have COVID-19. The communication reinforced the expectation that clerkship students would be involved in the care of such patients in a manner consistent with their level of training and dependent upon the availability of PPE.
Within just a couple of days, the situation nationally and locally further escalated. More restrictive social distancing was increasingly recommended and adopted. Our university encouraged working from home for those who could. Local districts began shuttering their schools. The hospitals where our students rotate also began serious preparations for an anticipated influx of patients with COVID-19: non-urgent or elective surgeries were postponed; PPE preservation initiatives were implemented; patient discharges were expedited when possible. Most outpatient clinics moved quickly to telehealth care delivery and postponed non-urgent visits. Hospital units began restricting access to visitors and volunteers. Several services changed their staffing models to reduce the number of faculty and residents working onsite in order to ensure a sufficient backup workforce should any staff members fall ill. Many faculty at our institution were heavily involved in contingency planning and ensuring operational readiness thereby limiting their ability to mentor and teach students.

CDs at our institution began to hear from students and supervisors alike that the rapidly shifting processes, policies, and priorities at many of our clinical sites were affecting the clinical learning environment. CDs met again in an urgent ad hoc teleconference on Friday, March 13, to discuss the new developments. Our discussion focused on two main themes and questions: (1) educational experience - were students still able to meet clerkship learning objectives? And (2) student, patient, and public health/safety - did continuing the clerkships create unacceptable risks to students and patients and/ or compromise the ability of healthcare teams to care for patients safely?

\section{Educational Experience}

CDs in a variety of disciplines noted that students were increasingly marginalized from clinical experiences. In part, this was due to the shifting focus of clinical services toward immediate operational readiness, often at the expense of the undergraduate medical education mission. Though students had been called "essential" by the medical school a week prior, several rotation sites began to raise concerns about student presence or restrict student access so as to conserve PPE and to limit transmission of the virus. As clinic-based physicians began to shift toward telehealth, incorporation of an off-site student into those online clinical encounters was difficult if not impossible in the short term.

The quality of the educational experience for students was also decreasing. On the surgical clerkships, few of any nonurgent procedures were being performed, and students were increasingly excluded from the operating room to conserve PPE. The same limitations on student use of PPE also rendered students unable to care for patients on contact/droplet 
precautions for reasons other than COVID-19, limiting the scope of their clinical exposure in, for example, Internal Medicine and Pediatrics. Restriction of access to units affected educational experiences including access to interview tutorials and physical exam training.

The availability of appropriate supervision had also become less reliable. Many faculty and residents had to reallocate time that would normally have been dedicated to student education to COVID-19 preparations. Changes to staffing models meant that on certain services, there were fewer faculty and residents on site to supervise students. Because of closed schools, some faculty and residents now had additional childcare responsibilities, so were less present to teach students. This shift in priorities away from student supervision seemed likely to continue as hospitals began to see an influx of patients with COVID-19.

Adequate administrative resources to orchestrate the clerkships were also quickly being challenged by crisis. Like other faculty, many CDs themselves were increasingly occupied by abrupt changes to their primary clinical and personal obligations. Identifying alternative rotation sites to take the place of those which had barred students from participating as well as developing and/or vetting substitute learning activities required significant time which CDs did not have under these conditions.

Not every clerkship was equally affected: some outpatient primary care offices remained open and functioning relatively normally, for example. The PPE conservation considerations were a more immediate issue on inpatient medicine and surgical services than on psychiatric services.

\section{Student/Patient/Public Health}

In light of the threatened compromise of the educational experience, CDs revisited the risks and benefits of having clerkship students present in clinical spaces. A week earlier, the educational benefits to the students and service benefits to the clinical teams seemed to outweigh the risks to student health, patient health, and public health. But that calculus changed as the crisis became more acute.

Though the risk of severe illness or death to any individual medical student was thought to be low, it was not zero. Furthermore, medical students were potential vectors for the virus, even if asymptomatic. Because student work would have to be supervised, a patient being cared for by a student would necessarily have to encounter at least one additional provider (student), perhaps several times per day. As with any additional body, a student's presence would make appropriate social distancing on rounds or in the charting room more challenging. Furthermore, having students continue at clinical sites would also mean they were potential vectors from the hospital into the community and from the community into the hospital. The projected scarcity of PPE also meant that students would likely not have access to such equipment. In this new reality, CDs felt that the students' primary professional duty had shifted from caring for ill patients to practicing and modeling appropriate social distancing in hopes of flattening the epidemiologic curve.

Because of the novelty of the virus, clinical recommendations were still evolving and not always consistent. Some sites questioned when and whether students who recently had fever and/or respiratory symptoms could safely return to the clinical environment. These issues were further muddled by the lack of availability of testing.

\section{Decision}

Based on the above concerns, on March 13, the CDs unanimously agreed that all clerkships should be suspended. After a lengthy discussion, a suspension of 2 weeks (which would become the AAMC's minimum recommendation) was thought to be too short because the clinical situation was likely to still be worsening within that time frame. A longer suspension would also provide students with the opportunity to do meaningful activities in furtherance of their education, including preparing for and taking USMLE Step 1. Ultimately, the CDs recommended a pause of 6-8 weeks with opportunities to re-evaluate the situation in the interim to determine if that initial suspension would be adequate.

The recommendation from the CDs was conveyed to the Dean of the School of Medicine and the Department Chairs that same day by the Deputy Dean for Education. Initially, the Dean and Chairs expressed their eagerness to address the concerns raised by CDs so that students could continue to participate in their clinical training. Over the weekend, as the situation nationally and locally continued to evolve, each $\mathrm{CD}$ reached out directly to his/her respective Chair to share the specific concerns from each clerkship about the considerable barriers to student participation in patient care and the challenges to providing appropriate educational opportunities. On Monday, March 16, the Department Chairs and Dean reconvened to discuss this additional input from the CDs and others and decided to suspend all clerkships for 8 weeks.

\section{Future Directions}

Our clerkships were suspended with 2 weeks remaining in the first clerkship block. One major question became whether the block would be considered complete or whether students would need to make up the two remaining weeks of clinical experiences at a later time. CDs and the Associate Dean for Curriculum agreed that the guiding principle should be whether students had achieved the clerkships' learning objectives. 
CDs varied in their assessments, but ultimately agreed that uniformity would be important and that when clerkships were restarted, students would complete the remaining 2 weeks.

CDs discussed whether clerkship objectives could be met through alternative means like online cases or videos. Though we each knew of excellent online educational resources in our respective specialties, we also agreed that much of the online content targeted medical knowledge and was less useful in helping students to develop clinical skills or enhancing professional attitudes. Of additional concern was whether substituting virtual experiences in place of direct patient care would create the message that real patients are extraneous to learning how to be a physician. Ultimately, it was thought that online resources should be used to supplement past and future patient care experiences rather than being used in lieu of such direct care.

The suspension will inevitably require a re-arrangement of students' academic schedules moving forward. Even barring additional disruptions, students in the current core clerkship year will have their clerkships extend into the next calendar year, at the same time as the next class is scheduled to begin clerkships. We may have to modify the structure and length of core clerkships to ensure that all students have sufficient clinical exposure to meet learning objectives. Other considerations include how to minimize the impact this suspension will have on the MD-PhD students who are required to complete two clerkship blocks before entering their research years and on the MD-PhD students who returned from research to complete their remaining two clerkship blocks. The timing of USMLE Step examinations, electives, and sub-internships for the current clerkship class will also be shifted.

Because of the rapidity with which the clerkships were suspended, there was little time beforehand to coordinate alternatives for how students should spend their time during the pause. Consideration was given to online learning, preparation for USMLE Step 1, and non-clinical electives. Outside of their academic responsibilities, many students also independently volunteered to assist in the response including manning COVID-19 information phone lines for the public, telephone outreach to isolated older patients, and providing childcare and other supports for front-line health care workers.

We are still in the beginning stages of the COVID-19 outbreak and response. Like so much of life at present, how and when students return to their "normal life" of being on clerkships remains uncertain. As disruptive as this situation continues to be, the experience may offer opportunities for innovation including developing new ways to incorporate student education into telehealth and enhancing distance learning resources. It will also be incumbent upon clerkship directors and faculty to ensure that medical students, though temporarily removed from the clinical setting, understand just how important they are as future physicians.

\section{Compliance with Ethical Standards}

Conflict of Interest The authors declare that they have no conflict of interest.

Ethics Approval Not applicable

\section{Reference}

1. Association of American Medical Colleges. Important guidance for medical students on clinical rotations during the coronavirus (COVID-19) outbreak. 2020. https://www.aamc.org/ news-insights/press-releases/important-guidance-medicalstudents-clinical-rotations-during-coronavirus-covid-19outbreak. Accessed April 14, 2020.

Publisher's Note Springer Nature remains neutral with regard to jurisdictional claims in published maps and institutional affiliations. 